\title{
MOLECULAR AND GENETIC FEATURES OF COLORECTALCANCER IN YOUNG PATIENTS
}

\author{
Semyanikhina A.V., Pospekhova N.I. \\ Federal State Budgetary Institution «N.N. Blokhin National Medical Research Centre of \\ Oncology» of the Ministry of Health of the Russian Federation (N.N. Blokhin NMRCO), \\ Moscow, e-mail: alexandra_silina@mail.ru
}

\begin{abstract}
The literature review represents the current state of research on molecular and genetic features of colorectal cancer in young patients. A steady increase in the incidence of colorectal cancer among young patients has been noted over recent years. Along with hereditary factors determining a high risk of colon tumours in patients who are carriers of germinal mutations in the genes responsible for the development of syndrome pathology (such as Lynch syndrome, familial adenomatous polyposis, Peutz-Jeghers syndrome, etc.), molecular and genetic changes in the cells of the colonic epithelium define the distinctive clinical and morphological features, prognosis, and response to specific therapy in young patients with colorectal cancer. To date, the main pathogenetic pathways involved in the promotion of carcinogenesis in the gut epithelium tissue have been identified: chromosomal instability, microsatellite instability, epigenetic abnormalities includinghyper- or hypomethylation of the tumour genome. Despite the well-studied molecular carcinogenesis of colorectal cancer, there are still many gaps in theunderstanding of the nature of such malignant neoplasms in patients with the disease diagnosed at an early age.
\end{abstract}

Key words: colorectal cancer, young patients, chromosomal instability, microsatellite instability, abnormal methylation, mutations

INTRODUCTION. Colorectal cancer (CRC) remains one of the most widespread malignant neoplasms in the structure of cancer incidence both in male and female patients. There has been a decrease in the number of newly diagnosed cases of CRC in the last years mostly due to currently implemented screening programmes that are able to provide timely diagnostics followed by the successful treatment of pretumour pathology of the gut, especially in patients who are 50 years old or older. [1, 2]. Negative dynamics of CRC incidence is registered among patients of the middle age groups that screening programmes are not designated for[1]. According to the American Statistical Center of Epidemiology, 15\% of patients with CRC are diagnosed before the age of 50 with the average disease onset - 45.2 years old [1]. A preliminary prognosis based on modern tendencies showed that the incidence rate for colon and rectal cancer will increase by $90 \%$ and $124.2 \%$ by 2030 for people aged 20-34 years old and by $46 \%$ - for people aged 35-49 years old [1, 3].

Early-onset colorectal cancer (EOCRC) is a heterogeneous group of colon and rectum malignant neoplasms with the disease onset at the age of 50 and under [1,2]., There is no presently accepted opinion on the borderline between "young" and "senior" patients [3]. The majority of authors believe that 50 years old is a cut-off based ongeneral population colorectal cancer screeningprogrammes. However, the data still varies in different studies [3].

Just part of EOCRC cases can be explained by inherited predisposition due to germline mutations and/or can be classified as a family case of CRC. In the majority of cases, EOCRC is 
characterized by a molecular nature that still remains in the focus of an increasing number of scientific and clinical studies.

The aim of the study was to provide an updated informtion on the problem of molecular- and enetic peculiarities of colorectal cancer in young patients.

Risk factors for the development of EOCRC. The carcinogenesis of CRC is a multifactor and multistage processat each stage of that exogenous and endogenous risks factors associated with CRC can be involved. However, none of these factors should be considered specific for EOCRC [4]. Smoking and alcohol consumption, a diet with a high content of red meat and fast food, a hypodynamic lifestyle are generally acknowledged as risk factors for CRC. Diabetes mellitus increases the risk of the development of gut neoplasms by $38 \%$. Each $5^{\text {th }}$ excess in body mass index is associated with a 13-18\% increase in the risk of CRC [4]. Chronic inflammation and gastrointestinal dysbiosis are also considered to be triggers of oncogenesis in the colon epithelium.

Burdened family history is one of the important risk factors predisposed to CRC. For example, a first-degree relative (FDR) with CRC in the family increases the risk of the development of CRC by 2-3 times. Two second-degree relatives (SDR) or one FDR with CRC diagnosed at the age under 50 are associated with a three-fold and more increase in the risk of CRC [5]. Relatives with adenomatous polyps also enhance the empirical risk of CRC. The age of CRC onset in the family is another prognostic factor. Thus, if CRC is diagnosed at the age of 90, it determines a 1.5time increase in the risk of CRC, at the age of 50 - four-fold and more [5].

However, the majority of patients with EOCRC is characterized by the absence of family cases of CRC in the first and second generations [4], which is, on the whole, a specific feature of EOCRC. After the exclusion of family history factor along with exluding of inflammatory bowel diseases, the majority of patients with EOCRC could be included in the cohort of patients with the general population risk of CRC [4].

In turn, negative family history does not mean that the disease is not inherited or a patient with EOCRC is not a carrier of germline mutations [4]. Thus, less than a half of the patients with Lynch syndrome meets the "Amsterdam" criteria; the pathological MSH6- and PMS2-genotype is characterized by incomplete penetrance; familial adenomatous polyposis (less common, Lynch syndrome) is associated with pathogenic germinal mutations de-novo in the APC gene in approximately $15 \%$ of all the cases. According to the data obtained by targeted sequencing of the germline genome, the rate of pathogenic mutations among patients with EOCRC is 15-20\%. According to Chang et al., at least $22 \%$ of CRC cases diagnosed in patients under 40 years old can be associated with hereditary syndromes [6]. A prospective study provided by Pearlman et al. showed that $16 \%$ of patients with EOCRC (72/450) have carried pathogenic mutations. At the same 
time, in $10 \%$ of the cases, germinal mutations were revealed in the genes with high penetrance (mismatch repair genes, $A P C, M U T Y H, B R C A 1 / 2, C D K N 2 A$, etc.), and in $6 \%$ of the cases - in the genes with intermediate risk of disease development (ATM, PALB, CHEK2, etc.) [7]. Meanwhile, a significant part of patients in such studies did not meet the criteria of the diagnosed syndrome either in clinical or in anamnestic aspects.

Despite the achieved success in molecular diagnostics, DNA-testing of germline genome has failed to detect any inherited cause and remains non-informative in the majority of EOCRC cases Adding to diagnostic panels candidate genes with intermediate or even low penetrance, could enrich the knowledge on the molecular nature of EOCRC.

Molecular pathogenesis of EOCRC. The carcinogenesis of CRC includes three main genetic mechanisms: chromosomal instability (CIN), microsatellite instability (MSI), and CpG island methylator phenotype (CIMP phenotype) [8]. The pathogenesis of EOCRC as part of syndromal pathology (Lynch syndrome, FAP, etc.) is a well-understood paradigm, while the identification of driver events in sporadic EOCRC has not yet been well studied. In general, EOCRC is characterized by a stable MSI phenotype (up to 80\%) [4], a low level of methylation and/or absence of CIMP, and euploidy of tumor cells.

MSI-High EOCRC. A high level of microsatellite instability can be detected in $21 \%$ of all the EOCRC cases and nearly in all of these positive cases, Lynch syndrome is subsequently proved by MMR-genotyping. Hypermethylation of the promoter region of the $M L H 1$ gene along with the presence or absence of somatic $B R A F$-mutations in young patients with MSI-High-CRC is quite a rare event ( $\mathrm{p}=0.006$ [4]). This combination was revealed only in two studies. Inn one studythe age of the patient was 49 years old $[9,10]$. Some degree of chromosomal instability was revealed in approximately $50 \%$ of MSI-High-associated EOCRC, , which demonstrated molecular heterogeneity of MSI-High CRC [9].

Microsatellite stable (MSS)-CRC and chromosome unstable ways of carcinogenesis. CIN is the main pathogenic pathway in the carcinogenesis of EOCRC. It is revealed in 50-80\% of all the cases [11]. These tumors are characterized by an intact mismatch repair system, accumulation of chromosome mutations leading to the change in their number, as well as chromosome large rearrangements, amplification or loss resulting in oncogenes upregulation or tumour growth suppressor genes inactivation. For the most part, $W N T$ - and $M A P K$-signal pathways, the genes TP53, DCC, SMAD2/4, etc. are involved in the pathological process [12].

Chromosome imbalance in CRC tumours with late onset is mainly associated with the loss of chromosome loci that include the SMAD4, DCC and $A P C$ genes. In turn, EOCRC differs by the 
amplification of the regions that contain the BMPRIA and AMP genes, in addition to the loss of loci with the TJP2 genes and genes coding FOX-transformation factors [13].

Activating mutations in the KRAS gene are revealed in 35-40\% of CRC cases beingthe predictors of resistance to anti-EGFR- therapy. The rate of the KRAS pathological genotype in EOCRC and in CRC diagnosed after 50 years varies in different studies and depends on patients characteristics, tumour localization and stage at the moment of diagnosis [14, 15].

The somatic mutation V600E in the BRAF gene is detected four times more often in patients with MSS-EOCRC than in patients with late-onset MSS-CRC (12\% vs 3\%, p<0.01) [16].

Whole-exome sequencing of tumour samples in different patient groups (under 45 years and older 65 years) did not reveal significant differences in the spectrum of genes with a high rate of mutations. In both cohorts, the rate of somatic mutations in the genes APC was $81.8 \%, T P 53-$ 74.6\%, KRAS - 46.4\%, ARIDIA - 19.4\%, PIK3CA - 13.4\%, FBXW7 - 11.2\%, SMAD4 - 10.1\%, $A T M-6.3 \%, N R A S-4.9 \%, B R A F-4.6 \%$ [3], while the somatic status of the genes responsible for hereditary CRC differed and was characterized by the prevalence of mutations in patients with EOCRC including the genes MSH6 (4.8\% vs $1.2 \%, \mathrm{p}=0.005)$, MSH2 (2.7\% vs $0.0 \%, \mathrm{p}=0.004$ ), POLE (1.6\% vs $0.0 \%, \mathrm{p}=0.008)$, NF1 (5.9\% vs $0.5 \%, \mathrm{p}<0.001)$, SMAD4 (14.3\% vs 8.3\%, $\mathrm{p}=0.024)$, PTEN (4.3\% vs 1.4\%, p=0.03), TSC1 (1.1\% vs 0.0\%, p=0.031), TSC2 (1.6\% vs $0.2 \%, \mathrm{p}=0.048$ ) [3]. In this study tumour mutation burden was also evaluated by the authors . A high level of mutations (TMB-High $\geq 17$ mutation/Mb) wass observed more often in patients with EOCRC $(9.7 \%$ vs $2.8 \%$, $\mathrm{p}<0.001)[3]$.

The gene expression pattern in tumour specimens of CRC also differs in patients with different age of the disease onset: hyperexpression of EGF, VEGF and classic WNT pathways is observed in young patients unlike CRC with late onset, which determines the potential effectiveness of anti-EGFR and anti-VEGF monoclonal antibodies while treating patients with metastatic EOCRC [17].

Microsatellite-stable and non-CIN EOCRC. Up to $63 \%$ cases of MSS-EOCRC are characterized by the absence of chromosomal instability [10]. CIN and MSI-negative tumors (CMN-CRC) represent an independent cluster of colorectal tumours with particular molecular and genetic alterations that determine their biological behavior and phenotypic characteristics of the tumour. For the most part, such tumours are localized in the rectum, appeared in patients with a burdened family history and diagnosed at the later stages. CMN-CRC is revealed significantly more often in younger patients than in older ones $(63.6 \%$ vs $12.5 \%, \mathrm{p}=0.005)$ and it is responsible for approximately $50 \%$ of colorectal cancer in patients under 50 years old [18, 19]. 
Despite the diploid karyotype, the features of chromosomal instability can be detected in some CMN-CRC tumours. However, in such a case, CIN is a very rare or very late pathogenic event. One of the distinguishing features of CMN-CRC, unlike CIN-associated CRC, is the suppression of apoptotic activity in CMN tumors due to telomerase-independent elongation of telomeres. At the same time, in tumours with chromosomal instability, shorter telomeres are formed due to the reactivation of telomerase $(\mathrm{p}=0.0066)$ [19].

Hypomethylation in EOCRC tumours. Hypomethylation is a frequent and early epigenetic event in colorectal carcinogenesis. It affects repetitive elements of nucleic acids in the tumour genome and can be detected by the methylation status of LINE1 (Long Interspersed Nuclear Element-1) [20, 21]. The studies with experimental models have revealed that the suppression of the expression of DNMT1 induces global DNA-hypomethylation, loss of heterozygosity in the $A P C$ containing locus and led to the initiation of adenoma growth in the colonic epithelium [21].

A high level of hypomethylation is one of the specific characteristic of EOCRC $(<0.0001)$. It is observed mor frequentin younger patients with colorectal tumors [9]. Regardless of the age, all patients with hypomethylated tumours tend to have family history burdened with CRC, such neoplasms affect distal parts of the colon and rectum $(\mathrm{p}=0.03)$, and are characterized by MMRsufficient phenotype in the most cases $(\mathrm{p}=0.004)[22,23]$. A higher rate of hypomethylation can be seen in tumours with a lack of chromosomal and microsatellite instabilities unlike CIN-CRC tumours $(p=0.004)$ [22]. Hypomethylation is an alternative pathogenic pathway that evolves into $\mathrm{CIN}$ at the later stages of carcinogenesis. Being quite a rare event, but cases of hypomethylation have also been registered in MSI-High tumours with diploid karyotype..

Clinical presentation, prognosis and outcome of EOCRC. Up to a point it was believed that EOCRC was characterized by a negative prognosis. However, recent studies have demonstrated the variability of clinical outcomes in young patients with CRC. Watson et al. in their study showed that the median overall survival(OS) in patients with EOCRC of stages III and IV was significantly higher than in patients with CRC diagnosed after 50 (34 vs 28 months, p=0.0145, and 30 vs 11 months, $\mathrm{p}<0.0001$, respectively) [14]. Another study presented the data on the 5-year OS of patients with stages II and IV and proved that patients with EOCRC had better long-term outcomes [24]. The prognostic difference of the age of onsetcan be explained by a comorbid background in older patients that limits therapeutic approaches, including surgery, chemotherapy, etc. Patients with EOCRC that underwent only surgical treatment demonstrate the best 5-year CRC-associated survival rate regardless of the disease stage and a lower rate of surgical mortality (0.7 vs $5.0 \%$, $\mathrm{p}=0.026$ ) [25]. Young patients are better ableto tolerate aggressive chemotherapy schemes. Some studies have showed that young patients are characterized by a higher therapeutic sensitivity, which 
in turn could explain a higher survival rate in patients with EOCRC at stage IV [25]. A high incidence of Lynch syndrome among young patients (15-20\%) also significantly improves statistics on the survival in this group of patients.

MSI-High CRC. Non-metastatic MSI-High-CRC is characterized by a favorable clinical prognosis. The later the disease is diagnosed the less positive effect MMR-deficient status provides. On the whole, young patients with MSI-High CRCdemonstrate a higher rate of OS $(\mathrm{p}=0.045)$ and more frequent appear to have the disease at the early stages in comparison with patients with MSSCRC (51.9 vs $29.7 \%, \mathrm{p}=0.03)$ [16].

CIN-EOCRC. Pathological tumour KRAS-genotype in patients with CIN- EOCRC is associated with a poorer prognosis in comparison with the patients with CRC carrying the wild type of this gene. The majority of $K R A S$-positive tumours in young patients are found in the distal colon and rectum (62\%) and diagnosed at III and IV stages (26\% and 63\%, respectively).

Activating mutations in the BRAF gene are proven negative prognostic markers for MSSEOCRCthat result inthe constitutional activation of $R A F$-kinase and an aggressive tumour phenotype [16]. CRC with $B R A F$-mutations is revealed at III and IV stages in up to $100 \%$ of cases, which provides poorer survival rates [16]. A5-year disease-specific survival (DSS) in patients with mutBRAF-EOCRC is only $16 \%$ [16].

CIN-and MSI-negative EOCRC. Regardless of the age of onset, a high metastatic potential of tumours without obvious features of CIN and MSI predicts early dissemination and an unfavorable prognosis $(\mathrm{HR}=2.44, \mathrm{p}=0.0004)$ [26]. The survival rates of patients with CMN-CRC are lower than such indicatorsof patients with CIN- or MSI-High-associated CRC ( $=0.0013)$. This fact is especially evident for patients with EOCRC [26].

CMN-CRC is less immunogenic in comparison with CIN- and/or MSI-associated tumours $(p<0.0001)$ [26]. Genome stability of CMN-CRC leads to a decrease in lymphocytic infiltration of the tumour and its microenvironment along with downregulation $\mathrm{n}$ of INF $\gamma$ - and IL18-expression. A lack or decrease in the production of superficial antigens allows CMN-CRC to avoid the immune response.

EOCRC with hypomethylation. Hypomethylation in patients with $\mathrm{CRC}$ is one of thenegative prognostic factors for CRC arising from the proximal colon. A low level of methylation correlates with the late stage of the disease at the moment of diagnosis $(\mathrm{p}=0.0001)$ and a 5-year OS of $48 \%$ ( $\mathrm{p}=0.02$ ) [23]. The molecular mechanism that underpins this negative influence still remains unclear. Hypomethylation of the genome leads to the increase in the expression of transcriptional proto-oncogenes (MET, etc.), chromosomal instability, activation of transposons, and induction of oxidative stress which independently or in the combination determine an aggressive clinical 
behaviour of the tumour and a negative prognosis [27]. Besides, tumours with a low level of methylation, as well as $\mathrm{CMN}-\mathrm{CRC}$, are characterized by decreased immunogenicity and a low level of tumour-infiltrating T-lymphocytes [28].

\section{CONCLUSIONS.}

A stable increase in the CRC incidence is observed among young patients. The increase is forecast to reach $142 \%$ by 2030 .

By now, no universal risk factors have been identified that could contribute to this dynamics among the young population.

Despite the diagnostics of the disease at later stages and aggressive clinical development, patients with MCRC have better long-term outcomes in comparison with older patients with CRC.

Once Lynch syndrome is suspected, microsatellite instability testing should be regarded when possible, followed by MMR-genotyping in case of MSI-High. Bypass MSI-test when a tumour sample is not available. . If clinical and anamnestic signs of another hereditary syndrome are appeared while consulting a patient with EOCRC DNAgenotyping may be proposed at the first diagnostic stage.

Just in 15-20\% cases EOCRC is associated with microsatellite instability and, in the majority of cases - with chromosomal instability.

Hypomethylation is is one of the significant mechanisms of the carcinogenesis of colorectal cancer and an important independent factor of negative prognosis for the patients with EOCRC.

\section{FINANCIAL SUPPORT AND SPONSORSHIP}

Nil.

\section{CONFLICTS OF INTEREST}

The authors declare no conflict of interest

\section{SUPPLEMENTARY DATA (DOI)}

\section{REFERENCES}

1. Chen F.W., Sundaram V., Chew T.A et al. Advanced-stage colorectal cancer in persons younger than 50 years not associated with longer duration of symptoms or time to diagnosis. Clinical Gastroenterology and Hepatology, 2017, vol. 15, no 5, pp. 728-737, doi 10.1016/j.cgh.2016.10.038. 
2. Patel S.G., Ahnen D.J. Colorectal Cancer in the Young. Current Gastroenterology Reports, 2018, vol. 20, no. 4, pp. 2-11, doi 10.1007/s11894-018-0618-9.

3. Puccini A., Lenz H.J., Marshall J.L. et al. Impact of Patient Age on Molecular Alterations of Left-Sided Colorectal Tumors. Oncologist, 2018, vol. 24, no 3, pp. 319-326, doi 10.1634/theoncologist.2018-0117.

4. Cavestro G.M., Mannucci A., Zuppardo R.A. et al. Early onset sporadic colorectal cancer: Worrisome trends and oncogenic features. Digestive and Liver Disease, 2018, vol. 50, no. 6, pp. 521-532, doi 10.1016/j.dld.2018.02.009

5. Burt R.W. Inheritance of Colorectal Cancer, Drug Discovery Today: Disease, 2007, vol. 4, no. 4, pp. 293-300, doi 10.1016/j.ddmec.2008.05.004.

6. Chang D.T., Pai R.K., Rybicki L.A. et al. Clinicopathologic and molecular features of sporadic early-onset colorectal adenocarcinoma: an adenocarcinoma with frequent signet ring cell differentiation, rectal and sigmoid involvement, and adverse morphologic features. Modern Pathology, 2012, vol. 25, no 8, pp. 1128-1139, doi 10.1038/modpathol.2012.61.

7. Pearlman R., Frankel W.L., Swanson B. et al. Ohio Colorectal Cancer Prevention Initiative Study Group. Prevalence and spectrum of germline cancer susceptibility gene mutations among patients with early-onset colorectal cancer. JAMA Oncology, 2017, vol. 3, pp. 464-471, doi 10.1001/jamaoncol.2016.5194.

8. Grady W.M., Markowitz S.D. The molecular pathogenesis of colorectal cancer and its potential application to colorectal cancer screening. Digestive Diseases and Sciences, 2015, vol. 60, no 3, pp. 762-772, doi 10.1007/s10620-014-3444-4.

9. Antelo M., Balaguer F., Shia J. et al. A high degree of LINE-1 hypomethylation is a unique feature of early-onset colorectal cancer. PLoS One, 2012, vol. 7, no 9, p. e45357, doi 10.1371/journal.pone.0045357.

10. Losi L., Gregorio C.Di, Pedroni M. et al. Molecular genetic alterations and clinical features in early-onset colorectal carcinomas and their role for the recognition of hereditary cancer syndromes. The American Journal of Gastroenterology, 2005, vol. 100, no 10, pp. 2280-2287, doi 10.1111/j.1572-0241.2005.00223.x.

11. Bardhan K., Liu K. Epigenetics and colorectal cancer pathogenesis. Cancers, 2013, vol. 5, pp. 676-713, doi 10.3390/cancers5020676.

12. Arriba M., García J.L., Inglada-Pérez L. at al. DNA copy number profiling reveals different patterns of chromosomal instability within colorectal cancer according to the age of onset. Molecular Carcinogenesis, 2015, vol. 55, no 5, pp. 705-716, doi 10.1002/mc.22315.

13. Berg M., Agesen T.H., Thiis-Evensen E. et al. Distinct high resolution genome profiles of early onset and late onset colorectal cancer integrated with gene expression data identify candidate susceptibility loci. Molecular Cancer, 2010, vol. 9, no 1, p. 100, doi 10.1186/1476-4598-9-100. 
14. Watson R., Liu T.-C., Ruzinova M.B. High frequency of KRAS mutation in early onset colorectal adenocarcinoma: implications for pathogenesis. Human Pathology, 2016, vol. 56, pp. 163-170, doi 10.1016/j.humpath.2016.06.010.

15. Yantiss R.K., Goodarzi M., Zhou X.K. et al. Clinical, pathologic, and molecular features of early-onset colorectal carcinoma. The American Journal of Surgical Pathology, 2009, vol. 33, no 4, pp. 572-582, doi 10.1097/pas.0b013e31818afd6b.

16. Khan S.A., Morris M., Idrees K. et al. Colorectal cancer in the very young: a comparative study of tumor markers, pathology and survival in early onset and adult onset patients. Journal of Pediatric Surgery, 2016, vol. 51, no 11, pp. 1812-1817, doi 10.1016/j.jpedsurg.2016.07.015.

17. Laurent-Puig P., Manceau G., Zucman- Rossi J. et al. Dual blockade of epidermal growth factor receptor-induced pathways: a new avenue to treat metastatic colorectal cancer. Journal of Clinical Oncology, 2012, vol. 30, no 13, pp. 1550-1552, doi 10.1200/jco.2011.40.1190.

18. Chan L., Curtis L.C., Leung S.Y. at al. Early-onset colorectal cancer with stable microsatellite DNA and near-diploid chromosomes. Oncogene, 2001, vol. 20, no 35, pp. 4871-4876, doi 10.1038/sj.onc.1204653.

19. Boardman L.A., Johnson R.A., Petersen G.M. at al. Higher frequency of diploidy in youngonset microsatellite-stable colorectal cancer. Clinical Cancer Research, 2007, vol. 13, no 8, pp. 2323-2328, doi 10.1158/1078-0432.ccr-06-2739.

20. Suzuki K., Suzuki I., Leodolter A. et al. Global DNA demethylation in gastrointestinal cancer is age dependent and precedes genomic damage. Cancer Cell, 2006, vol. 9, no 3, pp. 199-207, doi 10.1016/j.ccr.2006.02.016.

21. Sheaffer K.L., Elliott E.N., Kaestner K.H. DNA hypomethylation contributes to genomic instability and intestinal cancer initiation. Cancer Prevention Research, 2016, vol. 9, no 7, pp. 534546, doi 10.1158/1940-6207.capr-15-0349.

22. Ogino S., Nishihara R., Lochhead P. et al. Prospective study of family history and colorectal cancer risk by tumor LINE-1 methylation level. Journal of the National Cancer Institute, 2013, vol. 105, no 2, pp. 130-140.

23. Mima K., Nowak J.A., Qian Z.R. et al. Tumor LINE-1 methylation level and colorectal cancer location in relation to patient survival. Oncotarget, 2016, vol. 7, no 34, pp. 55098-55109, doi 10.18632/oncotarget.10398.

24. O’Connell J.B., Maggard M.A., Liu J.H. et al. Do young colon cancer patients have worse outcomes? World Journal of Surgery, 2004, vol. 28, no 6, pp. 558-562, doi 10.1007/s00268-0047306-7.

25. Liang J.-T., Huang K.-C., Cheng Y.-M. et al. P53 overexpression predicts poor chemosensitivity to high-dose 5fluorouracil plus leucovorin chemotherapy for stage IV colorectal 
cancers after palliative bowel resection. International Journal of Cancer, 2002, vol. 97, no 4, pp. 451-457, doi 10.1002/ijc.1637.

26. Banerjea A., Hands R.E., Powar M.P. et al. Microsatellite and chromosomal stable colorectal cancers demonstrate poor immunogenicity and early disease recurrence. Colorectal Disease, 2009, vol. 11, no 6, pp. 601-608, doi 10.1111/j.1463-1318.2008.01639.x.

27. Jones P.A., Baylin S.B. The epigenomics of cancer. Cell. 2007, vol. 128, no 4, pp. 683-692, 10.1016/j.cell.2007.01.029.

28. Nosho K., Baba Y., Tanaka N. et al. Tumour-infiltrating T-cell subsets, molecular changes in colorectal cancer, and prognosis: cohort study and literature review. The Journal of Pathology, 2010, vol. 222, no 4, pp. 350-366, doi 10.1002/path.2774. 